\title{
Restructuring of plasmonic nanoparticle aggregates with arbitrary particle size distribution in pulsed laser fields
}

\author{
A E Ershov ${ }^{1,3,4}$, A P Gavrilyuk ${ }^{1,3}$, S V Karpov ${ }^{2,3,4, \dagger}$, and S P Polyutov ${ }^{3}$ \\ 1 Institute of Computational Modeling, Russian Academy of Sciences, Krasnoyarsk 660036, Russia \\ ${ }^{2}$ L. V. Kirensky Institute of Physics of the Russian Academy of Sciences, Krasnoyarsk 660036, Russia \\ ${ }^{3}$ Siberian Federal University, Krasnoyarsk 660028, Russia \\ ${ }^{4}$ Siberian State Aerospace University, Krasnoyarsk 660037, Russia
}

(Received 11 April 2016; revised manuscript received 6 July 2016; published online 28 September 2016)

\begin{abstract}
We have studied processes of interaction of pulsed laser radiation with resonant groups of plasmonic nanoparticles (resonant domains) in large colloidal nanoparticle aggregates having different interparticle gaps and particle size distributions. These processes are responsible for the origin of nonlinear optical effects and photochromic reactions in multiparticle aggregates. To describe photo-induced transformations in resonant domains and alterations in their absorption spectra remaining after the pulse action, we introduce the factor of spectral photomodification. Based on calculation of changes in thermodynamic, mechanical, and optical characteristics of the domains, the histograms of the spectrum photomodification factor have been obtained for various interparticle gaps, an average particle size, and the degree of polydispersity. Variations in spectra have been analyzed depending on the intensity of laser radiation and various combinations of size characteristics of domains. The obtained results can be used to predict manifestation of photochromic effects in composite materials containing different plasmonic nanoparticle aggregates in pulsed laser fields.
\end{abstract}

Keywords: optodynamics, nanoparticle, surface plasmon, laser radiation

PACS: 78.67.Sc, 73.20.Mf

\section{Introduction}

The growing interest in various applications of plasmonic materials is demonstrated in current publications. Nanoplasmonics has a great potential and involves a wide range of research and application problems from nanofabrication of logic elements for perspective types of optical processors to nanosensorics and novel methods in medicine and biotechnology, ${ }^{[1-27]}$ just to name a few. Experimental studies on interaction of laser radiation with plasmonic nanoparticles and their aggregates include various nonlinear optical processes in metal nanocolloids with different aggregation states, photochromic reactions underlying the optical memory effect in the metal-dielectric nanocomposite, surface enhancement of Raman scattering in media containing plasmonic nanoaggregates, biomedical applications in theranostics, et al. Irradiation of plasmonic materials containing nanoparticles of noble metals by pulsed laser radiation is accompanied by variation of their optical properties and appearance of the nonlinear optical responses. The manifestation of these effects depends not only on the light intensity but also on the mean particle size and the degree of polydispersity.

The mechanisms of these processes remained unclear until the optodynamic model was developed, ${ }^{[26,27]}$ in which alterations of optical properties of domains were attributed to light-induced changes in their structures.

The effect of laser-induced spectral- and polarization-
DOI: $10.1088 / 1674-1056 / 25 / 11 / 117806$ selective transparency of aggregated Ag plasmonic nanocolloids was first observed in pico- and nano-second pulsed laser radiation ${ }^{[28]}$ and experimentally investigated in more detail in Refs. [29]-[31]. This effect is observed when the laser radiation frequency falls within the spectral range of the surface plasmon (see data and references in Refs. [28]-[31]). If the intensity of the laser radiation is optimum, there appears a long-lived and relatively narrow dip $(\leq 70-100 \mathrm{~nm})$ at the laser radiation frequency in the inhomogeneously broadened plasmonic absorption spectrum of the disordered colloidal aggregates (for $\mathrm{Ag}$ multiparticle aggregates, the width of the plasmonic absorption band can be over $700 \mathrm{~nm}$ ). The spectral width of the dip corresponds to the homogeneous absorption band of a single particle. The irradiated area in nanocomposites containing large colloidal Ag aggregates acquires the color and polarization corresponding to the incident radiation, which demonstrates the photochromic effect. The effect is attributed to the local photomodification of the aggregates (restructuring of their resonant domains).

Resonant domains in aggregates may consist of both individual particles and multiparticle groups of closely spaced plasmonic particles interacting in an optical field with each other at a certain frequency. These optical interactions affect the resonant spectral characteristics of the particles. The resonant frequency of the domain depends on the interparticle gaps, the size of the particles, and the geometry of the nearest 
surroundings of each particle by other particles. The particle interactions change the local optical field. The inhomogeneously broadened extinction spectrum of a multiparticle aggregate is a set of resonant bands distributed over a wide spectral range and corresponding to different resonant domains in the aggregate. ${ }^{[32]}$ If the geometry of the local environment of particles in a domain changes under the influence of the incident laser radiation, then the spectral characteristics of the domain are also simultaneously modified. The kinetics of the spectral characteristics of a simplest Ag particle domain undergoing photomodification in pulsed laser fields was studied in Refs. [26], [27], and [33]. The examples of dimers and trimers consisting of Ag nanoparticles of different sizes were shown in Ref. [33]. Spectral and polarization selectivity of the dip vanishes when the radiation intensity goes beyond the limit ${ }^{[28-31]}$ and affects the non-resonant domains. In this case, the spectral width of the dips significantly grows.

Alteration of resonant properties of a domain (frequency shift of the maximum in its extinction spectrum during the laser pulse) may be due to both rearrangement of the relative positions of neighboring particles and changes in the size, shape, and phase state (melting or evaporation of particles). The possibility of light-induced shift of particles in aggregates is discussed in Refs. [34]-[36]. Approaching of irradiated particles in a domain is accompanied by the spectral shift of the surface plasmon resonances. Photomodification is typically confined to relatively small resonant domains and does not affect the large-scale geometry of multi-particle aggregates.

References [26] and [27] described the models of interaction of pulsed laser radiation with multiparticle aggregates of metal nanoparticles. In Ref. [27], we described an improved optodynamic model, which in contrast to that in Ref. [26], takes into account polydispersity of nanoparticles and temperature dependence of the relaxation constant of the particle electron subsystem. Optical interparticle interactions ${ }^{[27]}$ are realized within the dipole approximation when the dipole moment of each particle is calculated by the coupled dipoles method. ${ }^{[37]}$ To improve the accuracy of the dipole approximation, we used a renormalization parameter ${ }^{[37,38]}$ to take into account the contribution of higher multipoles ${ }^{[38]}$ at shorter distances.

One of the most important problems in the experimental realization of photochromic reactions is to find the conditions when these spectral changes are maximal and at the same time they preserve the spectral selectivity. The latter concerns the application of plasmonic nanocomposite materials for optical data storage. For example, reference [30] reported the record of five narrow dips within a wide plasmonic absorption band at different laser radiation wavelengths in one irradiated spot of nanocomposite containing Ag colloidal aggregates.

In contrast, a higher light intensity threshold of spectral change is preferable in systems for waveguiding of modulated optical radiation such as periodical chains of plasmonic nanoparticles with a locally excited particle at the terminal of a chain. ${ }^{[39]}$ The above discussion motivates one to study photoinduced spectral changes in such systems as resonant domains of multiparticle aggregates for different particle sizes and radiation intensities.

We emphasize that the data obtained on laser-induced processes in resonant domains consisting of a limited number of particles make it possible to explain the photochromic and nonlinear optical processes experimentally observed in multiparticle colloidal aggregates comprising hundreds and thousands particles. Simulations of such processes in large colloidal aggregates are limited by the computer speed.

The model in Ref. [27] is able to give a clear interpretation of the previously inexplicable nonlinear optical effects. In particular, the sign of nonlinear refraction of Ag nanocolloids depends on the aggregation state and a number of particles in aggregates. ${ }^{[40,41]}$

In our paper, we carried out a numerical study based on the developed theoretical models. The obtained results allow ones to estimate whether the polydispersity of colloidal aggregates typical for experimental nanocolloids makes them unsuitable for photochromic media and whether this factor impairs the spectral selectivity of the photochromic reactions.

We do not study the associated processes, such as the change of the particle shapes in the laser field as the origin of selective changes in the plasmon absorption spectrum, just because the shifted particles after irradiation preserve the spherical shape and the spectral manifestation of shifts matches with the experimental data observed at significantly lower radiation intensity. Afterwards, we do not consider modification of single particles (such as the change of their shape) in high intensity laser fields ${ }^{[42,43]}$ because it is beyond the scope of this paper.

Note that the photochromic effects are not only observed in colloidal aggregates and irradiated by pulsed laser radiation but are also found in ablative aggregates. Such aggregates are composed of nanoparticles deposited on a dielectric substrate in a noble gas medium at low pressure under laser irradiation of a metal bulk target. ${ }^{[44]}$ In this case, a significant contribution to the emergence of a spectral dip in absorption spectra of the aggregate can be caused by melting and coalescence of initially quasi-spherical particles in the resonance domains. Such coalescence may lead to the formation of both very large particles and particles having a shape elongated along the direction of polarization.

The goal of our paper is to determine the optimal conditions for photomodification of resonant domains (using Ag dimer as an example) in plasmonic colloidal aggregates of spherical nanoparticles depending on the degree of their poly- 
dispersity, average particle size, particle size distribution, and interparticle gaps for arbitrary combinations of these parameters for different light intensities. Under these conditions, radiation does not significantly impair the spectral selectivity of the photochromic effect.

\section{Theory}

Full description of the model of resonant interaction of domains with pulsed laser radiation is given in Ref. [27] and basic equations are available in Ref. [43]. The model takes into account a wide range of interrelated mechanical, thermodynamic, chemical, physical, and optical processes. On the one hand, these processes are due to local interparticle interactions of various origins occurring in domains of a multiparticle aggregate and, on the other hand, they are due to the factors accompanying these interactions.

Particle movement in a domain of an aggregate restructured under applied laser pulse is calculated taking into account the van der Waals attraction of particles and their elastic repulsion due to deformation of polymer adsorption layers (adlayers) of the particles in the contact area, dissipative forces of viscous and contact interparticle friction as well as light-induced optical forces in the external optical field.

The model takes into account heating and melting of the metallic core of particles and of their polymer adsorption layers, and heat exchange between electron and ion components of the particle material as well as heat exchange with the interparticle medium. Furthermore, we take into account that heating and melting of the particle metal core increases the electron relaxation constant, which reduces the $Q$-factor of the particle surface plasmon resonance and, hence, changes the interaction of laser radiation with a resonance domain when the resonant properties of the system deteriorate. This explains the reason for dynamic spectral changes that manifest themselves only during the pulse action, which is particularly typical for picosecond pulses.

Our model is based on the system of differential equations and the set of linear algebraic equations from Ref. [27]:

$$
\begin{aligned}
\frac{\mathrm{d} \boldsymbol{r}_{i}}{\mathrm{~d} t}= & \boldsymbol{v}_{i}, \quad i=1, \ldots, N \\
m_{i} \frac{\mathrm{d} \boldsymbol{v}_{i}}{\mathrm{~d} t}= & \left(\boldsymbol{F}_{\mathrm{vdw}}\right)_{i}+\left(\boldsymbol{F}_{\mathrm{el}}\right)_{i}+\left(\boldsymbol{F}_{\mathrm{opt}}\right)_{i}+\left(\boldsymbol{F}_{\mathrm{f}}\right)_{i}, \\
\boldsymbol{F}_{\mathrm{vdw}}= & -\frac{\partial U_{\mathrm{vdw}}}{\partial \boldsymbol{r}}, \quad \boldsymbol{F}_{\mathrm{el}}=-\frac{\partial U_{\mathrm{el}}}{\partial \boldsymbol{r}}, \quad \boldsymbol{F}_{\mathrm{opt}}=-\frac{\partial U_{\mathrm{opt}}}{\partial \boldsymbol{r}}, \\
U_{\mathrm{opt}}= & -\frac{1}{4} \operatorname{Re} \sum_{i=1}^{N}\left[\boldsymbol{d}_{i} \cdot \boldsymbol{E}^{*}\left(\boldsymbol{r}_{i}\right)+\frac{1}{2} \boldsymbol{d}_{i} \cdot\left(\frac{\boldsymbol{d}_{i}}{\varepsilon_{0} \alpha_{i}}-\boldsymbol{E}\left(\boldsymbol{r}_{i}\right)\right)^{*}\right. \\
& \left.-\varepsilon_{0} \alpha_{i}\left|\boldsymbol{E}_{0}\right|^{2}\right], \\
d_{i \beta}= & \alpha_{i}\left[\left(E_{0}\right)_{\beta} \exp \left(\mathrm{i} \boldsymbol{k} \boldsymbol{r}_{i}\right)+\sum_{j \neq i}^{N} \sum_{\gamma=1}^{3} G_{\beta \gamma}\left(\boldsymbol{r}_{i j}^{\prime}\right) d_{j \gamma}\right]
\end{aligned}
$$

$$
\begin{aligned}
& \beta, \gamma=x, y, z \\
& \alpha_{i}=R_{i}^{3} \frac{\varepsilon_{i}-\varepsilon_{\mathrm{m}}}{\varepsilon_{i}+2 \varepsilon_{\mathrm{m}}-\frac{2}{3} \mathrm{i}\left(R_{i}|\boldsymbol{k}|\right)^{3}\left(\varepsilon_{i}-\varepsilon_{\mathrm{m}}\right)}, \\
& \sigma_{\mathrm{e}}=4 \pi|\boldsymbol{k}| \operatorname{Im}\left[\sum_{i=1}^{N} \frac{\boldsymbol{d}_{i} \cdot \boldsymbol{E}^{*}\left(\boldsymbol{r}_{i}\right)}{\left|\boldsymbol{E}_{0}\right|^{2}}\right], \quad Q_{\mathrm{e}}=\frac{\sigma_{\mathrm{e}}}{\sum_{i=1}^{N} \pi R_{i}^{2}}, \\
& \frac{\mathrm{d}\left(E_{\mathrm{el}}\right)_{i}}{\mathrm{~d} t}=-\frac{\left(E_{\mathrm{el}}\right)_{i}}{\tau_{\mathrm{r}}\left(\left(T_{\mathrm{m}}\right)_{i}\right)}, \quad \tau_{\mathrm{r}}=\tau_{0} \exp \left(\frac{U}{k_{\mathrm{B}} T_{\mathrm{m}}}\right), \\
& \left(C_{\mathrm{e}}\right)_{i} \frac{\mathrm{d}\left(T_{\mathrm{e}}\right)_{i}}{\mathrm{~d} t}=-g\left[\left(T_{\mathrm{e}}\right)_{i}-\left(T_{i}\right)_{i}\right]+\frac{W_{i}}{V_{i}}, \\
& W_{i}=\frac{\omega\left|\boldsymbol{d}_{i}\right|^{2}}{2 \varepsilon_{0}} \operatorname{Im}\left(\frac{1}{\alpha_{i}^{*}}\right), \\
& \frac{\mathrm{d}\left(Q_{\mathrm{i}}\right)_{i}}{\mathrm{~d} t}=g V_{i}\left[\left(T_{\mathrm{e}}\right)_{i}-\left(T_{\mathrm{i}}\right)_{i}\right]+\left(q_{1}\right)_{i} V_{i}, \\
& \left(T_{\mathrm{i}}\right)_{i}=\frac{\left(Q_{\mathrm{i}}\right)_{i}}{C_{i} V_{i}} H\left(\left(Q_{1}\right)_{i}-\left(Q_{\mathrm{i}}\right)_{i}\right) \\
& +\frac{\left(Q_{\mathrm{i}}\right)_{i}-\left(Q_{2}\right)_{i}}{C_{i} V_{i}} H\left(\left(Q_{1}\right)_{i}-\left(Q_{\mathrm{i}}\right)_{i}\right) \\
& +T_{\mathrm{L}}\left(R_{i}\right) \cdot H\left(\left(Q_{1}\right)_{i}-\left(Q_{\mathrm{i}}\right)_{i}\right) .
\end{aligned}
$$

Here $t$ is the time from the start of the pulse; $N$ is the number of particles, $m_{i}, R_{i}, \boldsymbol{v}_{i}, \boldsymbol{r}_{i}$, and $\boldsymbol{F}_{i}$ are the mass, radius, speed, radius vector of the center of mass of the $i$-th particle and the resultant force; $\left(\boldsymbol{F}_{\mathrm{vdw}}\right)_{i}$ is the van der Waals force, $\left(\boldsymbol{F}_{\mathrm{el}}\right)_{i}$ is the elastic force related to deformation of the adlayers of contacting particles; $\left(\boldsymbol{F}_{\text {opt }}\right)_{i}$ is the optical force caused by the interaction of light-induced dipoles; $\left(\boldsymbol{F}_{\mathrm{v}}\right)_{i}$ is the viscous friction force; $\left(\boldsymbol{F}_{\mathrm{f}}\right)_{i}$ is the tangential interparticle friction forces; $U_{\mathrm{opt}}$ is the potential energy of interaction of light-induced dipole moments; $\boldsymbol{d}_{i}$ is the light-induced dipole moment; $\boldsymbol{E}(\boldsymbol{r})$ is the strength of the external electromagnetic field ( $\boldsymbol{E}_{0}$ is the amplitude of field, the symbol $(*)$ denotes complex conjugation); $\alpha_{i}$ is the dipole polarizability of the $i$-th particle; $\tau$ is the laser pulse duration; $H(x)$ is the Heaviside function; $\boldsymbol{k}$ is the wave vector of the laser radiation; $\boldsymbol{r}_{i j}^{\prime}$ is the vector joining the centers of the particles, adjusted for the renormalization coefficient; $G_{\alpha \beta}$ is the interparticle interaction tensor; $\varepsilon_{i}$ is the dielectric constant of the particle taking into consideration its size, temperature, and aggregate state; $\varepsilon_{\mathrm{m}}$ is the dielectric constant of the interparticle medium; $\sigma_{\mathrm{e}}$ is the extinction cross section, $Q_{\mathrm{e}}$ is the extinction efficiency, $\left(E_{\mathrm{el}}\right)_{i}$ is the elasticity modulus of the particle adlayer; $\tau_{\mathrm{r}}$ is the relaxation time of molecular bonds in adlayers; $\left(T_{\mathrm{m}}\right)_{i}$ is the average temperature of the heated area near the $i$-th particle; $\left(T_{\mathrm{e}}\right)_{i}$ and $\left(T_{\mathrm{i}}\right)_{i}$ are the temperatures of the electron and lattice (ion) components of the $i$-th particle; $\left(C_{\mathrm{e}}\right)_{i}$ is the volumetric heat capacity of the electron components; $g$ is the rate of energy exchange between the electron and ion subsystems; $W_{i}$ is the absorbed power of the laser radiation; $V_{i}$ is the particle volume; $\omega$ is the angular frequency of the laser radiation; $\left(q_{1}\right)_{i}$ is the heat flow per unit volume describing thermal losses; $T_{\mathrm{L}}\left(R_{i}\right)$ is the size dependence of the melting temperature; $\left(Q_{\mathrm{i}}\right)_{i}$ is the thermal energy 
transferred to the ion component of a particle, $\left(Q_{1}\right)_{i}$ and $\left(Q_{2}\right)_{i}$ are the thermal energies at the initial and the final stages of melting. Greek subscripts denote the Cartesian components of vectors and tensors.

\section{Results and discussion}

We synthesized Ag hydrosols by applying chemical techniques described in Ref. [45] (using reduction of silver by $\mathrm{NaBH}_{4}$ ) and adding polyvinylpyrrolidone as the material for formation of nanoparticle adsorption layer and retarding agent to control the coagulation rate. One can see in Fig. 1(a) that nanoparticles in typical metal hydrosols have different sizes. The histogram of particle radius dispersion was obtained by processing 10 TEM images of nanoparticle aggregates in this hydrosol (Fig. 1(b)).
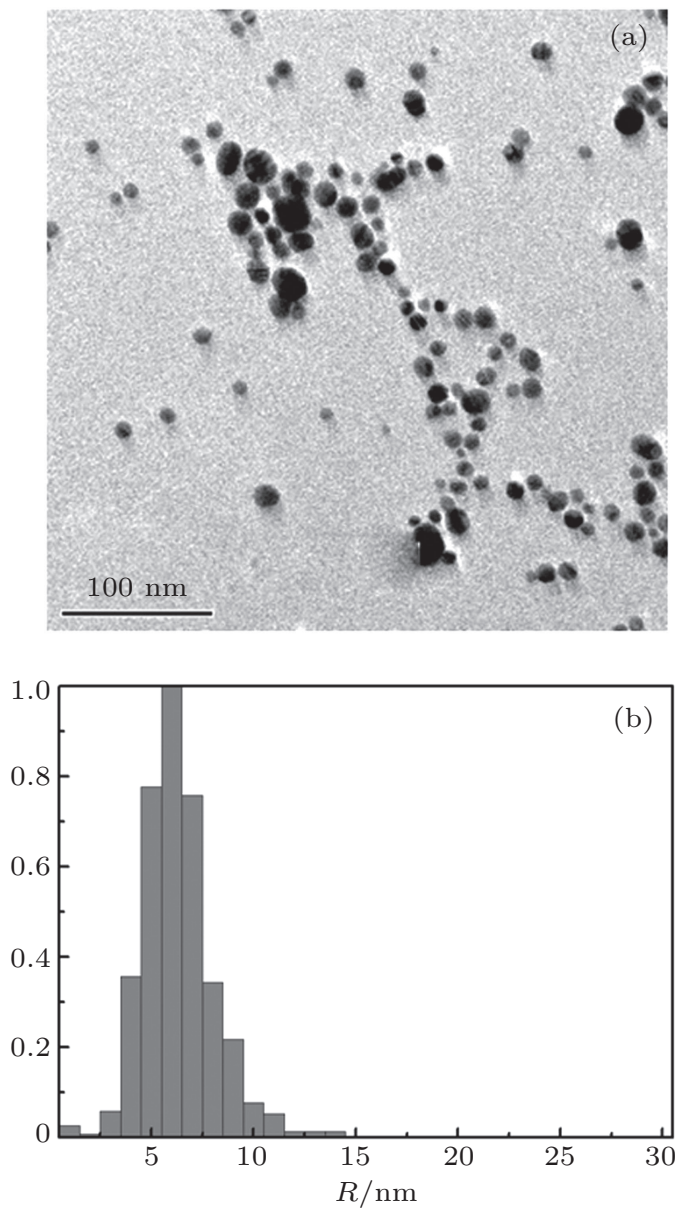

Fig. 1. (a) TEM image of polydisperse Ag nanoparticle aggregate of middle size in hydrosol and (b) its calculated histogram of particle radius dispersion averaged over a series of 10 TEM images.

Figure 2 shows the Ag nanoparticle aggregate generated by our aggregation model. ${ }^{[46,47]}$ This model is based on the molecular dynamics method. The positions of the groups of resonant particles in the aggregate (resonant domains consisting of various number of particles) at a certain wavelength are defined by the coupled dipoles method ${ }^{[27]}$ for two directions of radiation polarization. As one can see in the figure, there is a tendency to a collinear arrangement of resonant domains (hot points) relative to the polarization direction.

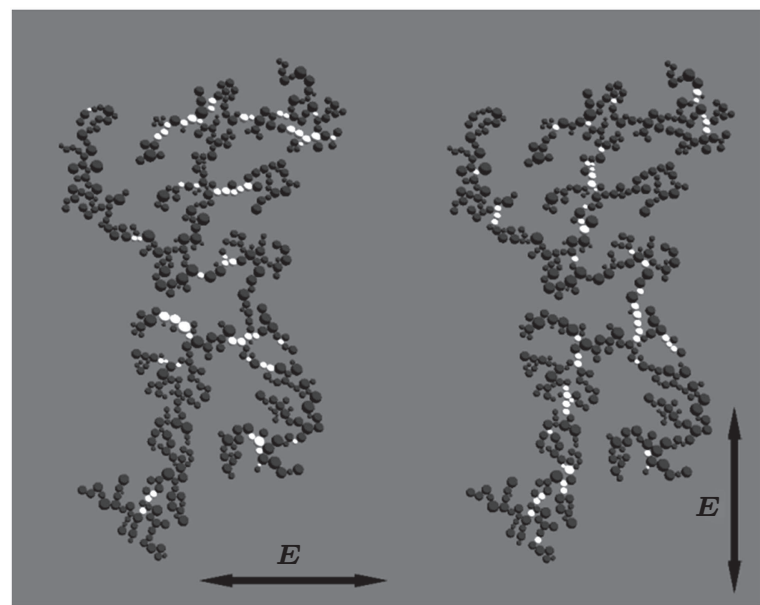

Fig. 2. Positions of resonant domains (shown in white color) in the simulated nanoparticle aggregate. These positions correspond to the ratio of the dipole moment of the particle to its volume exceeding 0.5 of the maximum magnitude of such ratio in the aggregate of $1000 \mathrm{Ag}$ nanoparticles. Particle size distribution is close to a truncated Gaussian function in the radius range $4-16 \mathrm{~nm}$. The radiation wavelength is $500 \mathrm{~nm}$. Polarization directions are indicated by the arrows.

Change in plasmonic absorption spectrum of multiparticle aggregate is the inherent manifestation of their photomodification. A typical example of such changes in our experiments is demonstrated in Fig. 3(a). This figure shows the formation of a spectrally-selective dip in the extinction spectrum of the aggregated Ag hydrosol (described above) exposed to nanosecond pulsed laser radiation. For aggregates of fractal type with low density, scattering is negligible in comparison with absorption, so the extinction spectrum is almost identical with the absorption one. The width of a dip is about $100 \mathrm{~nm}$ in the vicinity of the laser wavelength $(\lambda=532 \mathrm{~nm})$. Such width is close to the spectral band of surface plasmon resonance of a single particle. Figure 3(b) shows the extinction spectrum with the dip calculated using the model. ${ }^{[27]}$ The difference between these figures is as follows: figure 3(a) shows the experimental extinction spectrum with the dip in $10 \mathrm{~min}$ after the pulse end (pulse duration $\tau=15 \mathrm{~ns}$ ), and figure 3(b) shows the spectrum in $20 \mathrm{~ns}$ after the pulse is over $(\tau=20 \mathrm{ps})$. During this time, the particles with the adsorption layer can warm up and get closer to the interparticle gap, since the average time of approaching is 1-2 ns. The dip appears as a result of approaching of particles in the domain. Obviously, the experimental registration of this spectrum for such short time is impossible. In its turn, simulation time for a few tens of nanoseconds is equal to several hours. For simulation of 10 minute process, this time will be unrealistically long.

The width of a dip in the calculated spectral curve is somewhat larger than the experimental one, which may be due to limitations of the dipole approximation employed in 
the model and insufficient localization of the process induced by the laser pulse. However, taking into account the higher multipoles with fields localized near the particles in this type of models requires unrealistic computing power.
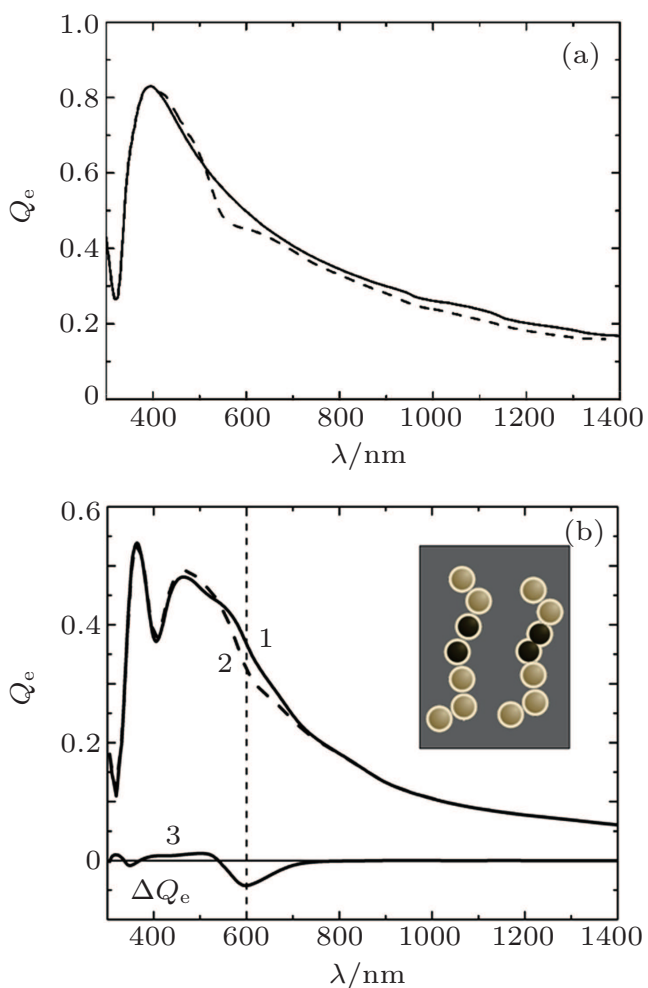

Fig. 3. (color online) Experimental (a) and calculated (b) extinction spectra of aggregated Ag hydrosol (within the surface plasmon absorption band) before and after irradiation and photomodification of aggregates (solid line) and after the end of laser pulse (dash line). Spectrum in panel (a) was obtained in 10 min, spectrum in panel (b) in $20 \mathrm{~ns}$ after the pulse. In panel (a), the pulse energy density is $8 \mathrm{~mJ} / \mathrm{cm}^{2}$, $\lambda=532 \mathrm{~nm}$, pulse duration $\tau=15 \mathrm{~ns}$, the number of pulses over a sample $1 \mathrm{~cm} \times 4 \mathrm{~cm}$ is 100 ; in panel (b), $\lambda=600 \mathrm{~nm}, \tau=20 \mathrm{ps}, 1-$ initial spectrum, 2 - spectrum after the pulse, 3 - the difference between spectra 1 and 2. The inset shows schematic draft of photomodification of two-particle dimer (marked dark) in the fragment of aggregate, showing the shift of particles in the dimer.

As was said above, laser-induced processes in resonant domains consisting of a limited number of particles underlie the photochromic and nonlinear optical processes in multiparticle colloidal aggregates. From this point of view, we can consider the case of a simple domain consisting of two Ag particles with the axis along the direction of radiation polarization to simulate light induced variations of its extinction spectrum. These variations can be significant and depend on the particle sizes, the initial interparticle distance, and the degree of polydispersity $\left(2\left|R_{1}-R_{2}\right| /\left(R_{1}+R_{2}\right)=\Delta R /\langle R\rangle\right)$. For a quantitative description of these changes, we introduce a spectrum photomodification factor

$$
\mu=\frac{\int_{0}^{\infty}\left|Q_{\mathrm{e}}(\lambda)-Q_{\mathrm{e} 0}(\lambda)\right| \mathrm{d} \lambda}{\int_{0}^{\infty} Q_{\mathrm{e} 0}(\lambda) \mathrm{d} \lambda},
$$

where $Q_{\mathrm{e} 0}(\lambda)$ is the extinction efficiency before modifications, and $Q_{\mathrm{e}}(\lambda)$ is the extinction efficiency at the end of modification $(0 \leq \mu \leq 2)$. The results of our calculations for an $\mathrm{Ag}$ dimer are shown in Fig. 4. This figure illustrates the initial and differential extinction spectra corresponding to various magnitudes of the photomodification factor for the Ag dimer irradiated by light of different intensities. We can see that the shift of particles to each other is accompanied by vanishing the initial absorption peak and its appearance in a longer wavelength range (dashed line).
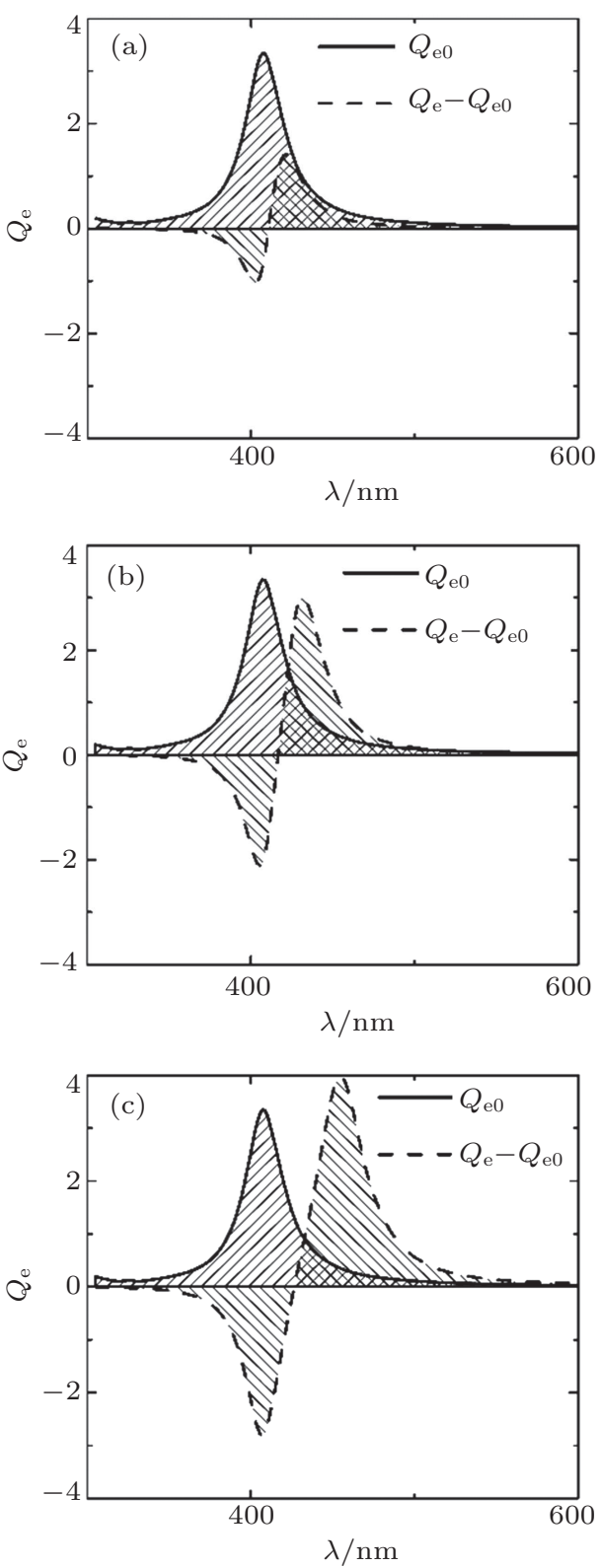

Fig. 4. Initial and differential extinction spectra of dimer $\left(R_{1}=R_{2}=\right.$ $5 \mathrm{~nm}$ ) corresponding to different magnitudes of photomodification factor $(\mu)$ : (a) -0.4 , (b) -1 , (c) -1.6 . Shading shows the numerator and denominator of Eq. (1).

As previously mentioned, the metal nanocolloids have different average particle sizes and the degree of particle polydispersity depends on different techniques of their synthesis. 
The question arises: how do these properties affect the interaction of the colloidal systems with laser radiation? Figure 5 shows the histograms of the photomodification factor depending on the average particle size and the degree of polydispersity. These histograms can predict the degree of manifestation of the laser photomodification in a colloidal system or in nanocomposite material containing Ag nanoparticle aggregates with an arbitrary particle size distribution in an optical field of a given intensity. The histograms show the magnitude of $\mu$ for each combination of the values of $\langle R\rangle$ and $\Delta R /\langle R\rangle$.

Note that even with the same initial thickness of the adlayer $(h)$, the interparticle gap $\left(h_{12}\right)$ varies depending on the particle size. For example, when $h=0.65 \mathrm{~nm}, h_{12}=$ $0.82 / 1.05 \mathrm{~nm} ; h=1.3 \mathrm{~nm}, h_{12}=1.82 / 2.15 \mathrm{~nm} ; h=1.8 \mathrm{~nm}$, $h_{12}=2.61 / 3.0 \mathrm{~nm}$. These variations are caused by the increased van der Waals attraction of the particles in the domain with larger particle sizes which, in its own turn, leads to greater compression of adlayers in the contact area (see discussion in Ref. [38])

For each dimer in calculations shown in Figs. 5(a)5(c), the laser radiation wavelength coincides with the maximum of the extinction spectrum for the radiation intensity $I=4.26 \times 10^{8} \mathrm{~W} / \mathrm{cm}^{2}$ typical for experiments with picosecond laser pulses. This is slightly below the threshold intensity corresponding to the onset of irreversible photomodification for monodisperse dimers with $R_{1}=R_{2}=5 \mathrm{~nm}$. For these conditions, photomodification does not occur $(\mu \rightarrow 0)$ for smaller average radii of particles and lower degree of polydispersity (the lower left corner in Fig. 5): the radiation intensity is below the threshold for such combinations of these parameters in dimers. Figures 5(d) and 5(e) show the histograms of the photomodification factor for lower radiation intensity $I=1.06 \times 10^{8} \mathrm{~W} / \mathrm{cm}^{2}$.

Figure 5 shows that the photomodification threshold is overcome when the interparticle gap $h_{12}$, the degree of particle polydispersity $(\Delta R /\langle R\rangle)$, and the average radius of the particles $(\langle R\rangle)$ grow. For example, for the monodisperse dimer $(\Delta R /\langle R\rangle=0)$ with an average particle radius $\langle R\rangle=6 \mathrm{~nm}$, photomodification does not occur, while for the polydisperse dimer $(\Delta R /\langle R\rangle=0.2)$ with the same average radius, photomodification takes place (Fig. 5(a)). The latter means that for the intensity of photomodification, the threshold for the monodisperse domain is above $4.26 \times 10^{8} \mathrm{~W} / \mathrm{cm}^{2}$, but for the polydisperse domain, it is below this level.

To explain the histograms, we should note that the photomodification associated with approaching of the particles in the domain is due to the decrease of the elasticity modulus of the particle adlayer under the action of heat (see. Eq. (7) in Ref. [33]). In turn, heating of the particles is determined by the amplitude of the light induced dipole moment of the particles (see. Eq. (8) in Ref. [33]). Thus, the photomodification thresh- old is associated with the efficiency of radiation absorption. Using these conclusions, we can explain the above-mentioned features.
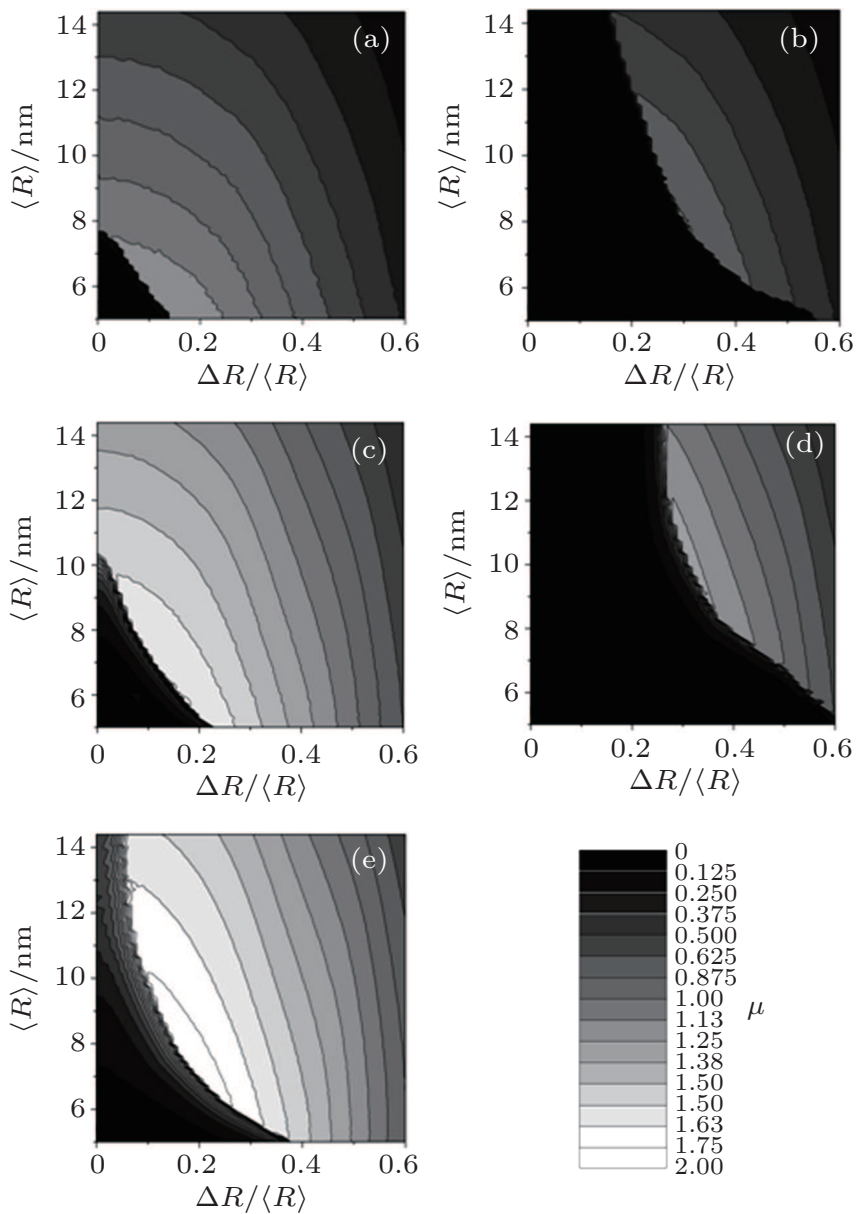

Fig. 5. Histograms of the photomodification factor $(\mu)$ of $\mathrm{Ag}$ dimer vs. the average particle size $\langle R\rangle$ and the degree of polydispersity for various thicknesses of the particle adlayer $h_{i}$ : (a), (d) $0.65 \mathrm{~nm}$; (b), (e) $1.3 \mathrm{~nm}$; (c) $1.8 \mathrm{~nm}$; light intensity $I$ : (a)-(c) $4.26 \times 10^{8} \mathrm{~W} / \mathrm{cm}^{2}$; (d), (e) $1.06 \times 10^{8} \mathrm{~W} / \mathrm{cm}^{2}$; pulse duration $\tau_{\mathrm{p}}=20 \mathrm{ps}$, in $20 \mathrm{~ns}$ after the pulse end. Tone scale corresponds to the values of $\mu$.

Since the dipole-dipole interaction is reduced in domains with larger interparticle gaps, this leads to a decrease of the local field produced by one particle near the other one. This results in a decrease of the amplitude of the dipole moment and, consequently, to a attenuation of the particle heating.

In the polydisperse dimer, the larger particle produces a larger local field in the vicinity of the smaller particle. Such a difference in the local field amplitude results in a relatively rapid heating of small particles and reduction of the elasticity modulus of the particle adlayer. Since the change of the elasticity modulus of the adlayer even of one particle in the pair is sufficient for photomodification, its threshold reduces upon increase of the particle polydispersity.

However, the further increase of the particle polydispersity reduces the photomodification factor. It can be explained by the reduced contribution of smaller particles to the extinction spectrum of the polydisperse dimer with domination of 
a large particle. The approach of a small particle to a large one changes the extinction spectrum of such a dimer insignificantly when compared to the case of the monodisperse dimer.

As remarked earlier, when the average radius of the particles increases, the strength of the van der Waals attraction grows, which leads to larger deformations of adlayers of these particles in the contact area. The approach of attracting particles leads to an increase of the local field produced by the particles on each other. This results in an increase of the absorbed radiation energy and greater heating of particles, which ultimately reduces the photomodification threshold.

The subsequent increase of the average particle radius reduces the interparticle initial gap relative to the distance between the centers of particles $\left(h_{12} /\left(R_{1}+R_{2}\right)\right)$. During photomodification, particles approach each other until the contact of metal cores, that means that the interparticle distance reduces to the initial interparticle gap. Consequently, when the relative change of the interparticle distance is the smaller, the radii of the particles will be larger. It results in a decrease of the photomodification factor $\mu$ when the average radius of the particles grows.

\section{Conclusion}

Nanocomposite materials and nanocolloids containing disordered locally anisotropic aggregates of plasmonic nanoparticles exposed to pulsed laser radiation exhibit the photochromic effect, allowing such materials to register the radiation characteristics: the wavelength and polarization in a wide wavelength range (450-1200 $\mathrm{nm}$ for silver). If the radiation intensity does not exceed the acceptable magnitude, the material bleaches in the irradiated area at the laser wavelength within spectral bandwidth $70-100 \mathrm{~nm}$. This bandwidth corresponds to the appearance of a dip in the extinction spectrum of the nanocomposite under the action of laser radiation. It makes it possible to record in the irradiated area (with a minimum size of the order of the light wavelength) the impact of radiation at several wavelengths and two orthogonal polarizations due to the photo-induced processes in the resonant domain of aggregates.

We have applied the optodynamic model to simulate the light-induced processes in the resonance domains of colloidal nanoparticle aggregates ${ }^{[27]}$ to a Ag nanoparticle dimer. This model allows one to study spectral photomodification of a resonant domain exposed to a pico- or nano-second laser pulse $\left(\tau_{\mathrm{p}}=20 \mathrm{ps}\right.$ ) depending on the particle size, the degree of polydispersity, and the interparticle gaps. We have obtained generalized two-dimensional histograms of distribution of the photomodification factor vs. the particle size distribution and the average particle size for various thicknesses of the particle adlayer.
We have shown that the best spectral selectivity of a dip (with minimum bandwidth) can be achieved using radiation of picosecond pulsed laser for nanocolloids or composite materials containing aggregates of monodisperse spherical particles with a mean radius about $10 \mathrm{~nm}$. In this case, the finite size effect and broadening of the particle absorption band are negligible, ${ }^{[1]}$ and at the same time the threshold intensity of laser radiation is low enough to prevent melting and deformation of particles.

Moderate polydispersity of nanocolloids $(\Delta R /\langle R\rangle<0.2)$ reduces the intensity threshold and does not significantly impair the spectral selectivity of the photochromic effect. Gradual decrease of the photomodification factor takes place upon further increase of the degree of polydispersity. Increase of the average particle size and interparticle gaps raises the intensity threshold required for manifestation of the photochromic effect.

The obtained results can be used to predict the tendency in spectral- and polarization-selective light-induced changes in plasmonic spectra of multiparticle polydisperse colloidal aggregates in pulsed laser fields for any combination of two parameters: the average particle size and the degree of particle polydispersity.

Besides the photochromic effect, the obtained results can be used to predict the amplitude of the nonlinear optical response in Ag nanocolloids in nanosecond laser pulses because light induced shift of particles in resonant domains and change of their optical properties occur for $1-2 \mathrm{~ns}^{[27,33]}$ that is within the pulse duration.

The applied optodynamic model is suitable for the study of interaction of high intensity optical radiation with nanoparticle aggregates with an arbitrary degree of structural ordering.

\section{Acknowledgement}

This work was performed within the state contract of the RF Ministry of Education and Science for Siberian Federal University for scientific research in 2015 (Reference number 1792) and SB RAS Program No II.2P (0358-2015-0010). Numerical computations were performed on the cluster MVS$1000 \mathrm{M}$ of the Institute of computational modeling SB RAS.

\section{References}

[1] Kreibig U and Vollmer M 1995 Optical Properties of Metal Clusters (Berlin: Springer)

[2] Stockman M I 2011 Opt. Express 1922029

[3] Shvets G and Tsukerman I 2011 Plasmonics and Plasmonic Metamaterials: Analysis and Applications (World Scientific Series in Nanoscience and Nanotechnology, Vol. 4)

[4] Shalaev V M 2000 Nonlinear Optics of Random Media: Fractal Composites and Metal-Dielectric Films (Berlin: Springer)

[5] Karpov S V and Slabko V V 2003 Optical and Photophysical Properties of Fractal-Structured Metallic Sols (Novosibirsk: Russian Academy of Sciences, Siberian Branch) 
[6] Stockman M I, Pandey L N and George T F 1998 Enhanced NonlinearOptical Responses of Disordered Clusters and Composites in Nonlinear Optical Materials (New York: Springer)

[7] Garcia M A 2011 J. Phys. D: Appl. Phys. 441

[8] Monticone F and Alù A 2014 Chin. Phys. B 23047809

[9] Li J B, He M D, Wang X J, Peng X F and Chen L Q 2014 Chin. Phys. B 23067302

[10] Tong L, Wei H, Zhang S, Li Z and Xu H 2013 Phys. Chem. Chem. Phys. 154100

[11] Jiang T T, Yin N Q, Liu L, Lei J M, Zhu L X and and Xu X L 2013 Chin. Phys. B 22126102

[12] Zolanvari A, Sadeghi H, Norouzi R and Ranjgar A 2013 Chin. Phys. Lett. 30096201

[13] Zolanvar A, Sadeghi H and Ranjgar A 2014 Chin. Phys. Lett. 31 106201

[14] Sadeghi H, Khalili H and Goodarzi M 2012 Chin. Phys. Lett. 29096201

[15] Zijlstra P and Orrit M 2011 Rep. Prog. Phys. 74106401

[16] Chen W W, Li T S, He S, Liu D B, Wang Z, Zhang W and Jiang X Y 2011 Science China - Chemistry 541227

[17] Wei A, Xiong L, Sun L, Liu Y J and Li W W 2013 Chin. Phys. Lett. 30 46202

[18] Ding D, Yang S E, Chen Y S, Gao X Y, Gu J H and Lu J X 2015 Acta Phys. Sin. 64248801 (in Chinese)

[19] Xiong Z C, Zhu L L, Liu C, Gao S M and Zhu J Q 2015 Acta Phys. Sin. 64247301 (in Chinese)

[20] Xu T N, Li X, Jia W W, Sui C H and Wu H Z 2015 Acta Phys. Sin. 64 245201 (in Chinese)

[21] Su D, Dou X M, Ding K, Wang H Y, Ni H Q, Niu Z C and Sun B Q 2015 Acta Phys. Sin. 64235201 (in Chinese)

[22] Rajwali K and Fang M H 2015 Chin. Phys. B 24127803

[23] Shen S C, Liu W T and Diao J J 2015 Chin. Phys. B 24127308

[24] Duan X Y and Wang Z G 2015 Chin. Phys. B 24118106

[25] Sun G L, Zhang L S and Hang L X 2013 Physics 42724

[26] Gavrilyuk A P and Karpov S V 2009 Appl. Phys. B 97163

[27] Ershov A E, Gavrilyuk A P, Karpov S V and Semina P N 2014 Appl. Phys. B 115547
[28] Karpov S V, Popov A K, Rautian S G, Safonov V P, Slabko V V, Shalaev V M and Shtokman M I 1988 JETP Lett. 48571

[29] Danilova Y E, Rautian S G and Safonov V P 1996 Bull. Russ. Acad. Sci. Phys. 60374

[30] Safonov V P, Shalaev V M, Markel V A, Danilova Y E, Lepeskin N N, Kim W, Rautian S G and Armstrong R L 1998 Phys. Rev. Lett. 801102

[31] Karpov S V, Slabko V V and Popov A K 2003 Tech. Phys. 48749

[32] Karpov S V, Bas'ko A L, Popov A K and Slabko V V 2000 Colloid. J. 62699

[33] Ershov A E, Gavrilyuk A P, Karpov S V and Semina P N 2015 Chin. Phys. B 24047804

[34] Drachev V P, Perminov S V, Rautian, S G, Safonov V P and Khaliullin E N 2002 J. Exp. Theor. Phys. 94901

[35] Drachev V P, Perminov S V and Rautian 2007 Opt. Express 158639

[36] Drachev V P, Perminov S V and Rautian 2008 Opt. Lett. 332998

[37] Markel V A, Shalaev V M, Stechel V B, Kim W and Armstrong P L 1996 Phys. Rev. B 532425

[38] Ershov A E, Isaev I L, Gavrilyuk A P, Karpov S V, Semina P N and Markel V A 2012 Phys. Rev. B 85045421

[39] Rasskazov I L, Karpov S V and Markel V A 2013 Opt. Lett. 384743

[40] Karpov S V, Kodirov M K, Ryasnyanskii A I and Slabko V V 2001 Quantum Electronics 31904

[41] Ganeev R A, Ryasnyansky A I, Kamalov S R, Kodirov M K and Usmanov T 2001 J. Phys. D: Appl. Phys. 341602

[42] Seifert G, Kaempfe M, Berg K J and Graener H 2001 Appl. Phys. B 73 355

[43] Yang G 2012 Laser Ablation in Liquids: Principles and Applications in the Preparation of Nanomaterials (New York: Pan Stanford Publishing)

[44] Plekhanov A I, Plotnikov G L and Safonov V P 1991 Opt. Spectrosc. 71451

[45] Heard S M, Griezer F and Rarraclough C G 1983 J. Colloid. Interface Sci. 93545

[46] Karpov S V, Gerasimov V S, Isaev I L and Obushchenko A V 2006 Colloid. J. 68441

[47] Karpov S V, Isaev I L, Gavrilyuk A P, Gerasimov V S and Grachev A S 2009 Colloid. J. 71313 